\title{
Multi-purpose materials in dentistry - is it possible to match maximum mechanical and biological properties/performance?
}

\author{
Dear Readers,
}

In this issue of the Journal of Applied Oral Science, two interesting studies raise an intriguing question: is it possible to match great mechanical and biological properties in multi-purpose materials used in dentistry?

In the first study, Caldeira, et al. ${ }^{1}$ (2013) investigate the antimicrobial and fluoride release ability of orthodontic bonding materials. Obviously, an efficient bonding is the primary clinical outcome expected from such kind of materials, but in the view of biofilm accumulation generated by orthodontic appliances, antimicrobial and enamel-protective properties also would be greatly desirable, as discussed by the authors. Interestingly, some of the bonding agents investigated presented satisfactory antimicrobial and fluoride release properties, but not all the materials presented the same efficacy in both tasks (in addition to an efficient bonding).

In a second report, Guven, et al. ${ }^{3}$ (2013) demonstrate that calcium silicate based endodontic cements may present some degree of toxicity over human tooth germ stem cells, when investigated in vitro simulating a biocompatibility challenge that could occur in periapical environment. As considered in the article, endodontic filling materials are expected to present efficient mechanical properties (i.e. provide an effective seal and be dimensionally stable), but ideally they should also be antimicrobial, biocompatible and pro-regenerative.

Drawing a rough parallel with our current daily routines, it is interesting to notice the coincidence between the recent proliferation of multi-purpose materials with the trend toward the execution of multiple/simultaneous tasks. Using some cognitive and neurobiology studies as example (an interesting reference is 'The Shallows', written by the 2011 Pulitzer Prize nominee Nicholas Carr ${ }^{2}$ ), it is becoming clear from recent studies that the execution of multiple simultaneous tasks compromise its excellence.

This interesting (and perhaps obvious) conclusion is to question if it is possible/viable to expect outstanding/ maximum mechanical and biological performance from multi-purpose materials, and how this expectation should guide clinical decisions.
In the dentistry context, it is important to remember that clinical framework of most materials (such as orthodontic bonding and endodontic cements) application is quite complex, and in vitro findings, while important in directing materials development, may not necessarily represent a clinical advance or advantage. Therefore, clinical studies based on the in vitro investigations are fundamental in determining if partially compromising mechanical properties of a given material to add some biological properties is clinically worthy, or if relatively simple additional procedures ('classic' biofilm control in the case of antimicrobial orthodontic bonding) could be equally (or even more) effective in promoting the clinical outcome expected. Alternatively, in clinical situations where a maximal mechanical performance is not necessarily fundamental for a successful clinical outcome, the partial exchange of mechanical to biological properties may be clinically relevant.

Therefore, it is very important to be aware of the possible advantages and disadvantages of the booming multi-purpose materials to be used in dentistry practice, developing specific in vitro analysis to test specific mechanical and biological aspects, in order to provide the basis for future clinical tests to support an effective application.

\section{References \\ 1- Caldeira EM, Osório A, Oberosler ELC, Vaitsman DS, Alviano DS, NOLIMA MCG. Antimicrobial and fluoride release capacity of orthodontic bonding materials. J Appl Oral Sci. 2013;21(4):327- 34. \\ 2- Carr N. The shallows: what the internet is doing to our brains. New York: W. W. Norton Co.; 2011. \\ 3- Güven EP, Yalvaç ME, Kayahan MB, Sunay H, Şahin F, Bayarli \\ G. Human tooth germ stem cell response to calcium-silicate based endodontic cements. J Appl Oral Sci. 2013;21(4):351-7.}

\author{
Gustavo Pompermaier Garlet \\ Co-Editor-in-Chief \\ Journal of Applied Oral Science
}

